ISAHP 1996, Vancouver, Canada, July 12-15, 1996

\title{
ON THE DERIVATION OF AHP PRIORITIES*
}

\author{
Jonathan Barzilai \\ School of Computer Science \\ Technical University of Nova Scotia, Halifax, N.S., Canada, B3J 2 X4 \\ barzilai@tuns.ca
}

\begin{abstract}
The geometric mean is the only acceptable solution to the problem of deriving weights from pairwise comparisons. Properties of acceptable solutions include immunity to rank reversals, independence of description of the problem, independence of scale inversion, uniqueness, independence of order of operations and inter-level consistency, preservation of the algebraic structure of the problem, extensibility to the additive case, related optimization models and related error measures.
\end{abstract}

\section{Introduction}

This paper is an overview of [2] where the issue of deriving priorities or weights in the Analytic Hierarchy Process (AHP) is analyzed. We refer the reader to that paper for proofs of theorems, numerical examples and detailed discussion.

\section{Structure and Notation}

\subsection{Conventions}

Throughout this paper, all matrices are $n \times n$; vectors are $n$-dimensional and vector and matrix operations apply componentwise.

\subsection{Definitions}

1. $A=\left(a_{i j}\right)$ is a pairwise multiplicative matrix if $0 \dot{<} a_{i j}=1 / a_{j i}$.

2. $w=\left(w_{k}\right)$ is a multiplicative weight vector if $w_{k}>0$ and $\prod_{k=1}^{n} w_{k}=1$.

3. $A=\left(a_{i j}\right)$ is a multiplicative consistent matrix if $a_{i j}=w_{i} / w_{j}$ for some multiplicative weight vector $w$.

4. $A^{\times}, w^{\times}$and $C^{\times}$are the sets of all pairwise multiplicative matrices, multiplicative weight vectors and multiplicative consistent matrices, respectively.

5. $f^{\times}$is the set of all mappings from $A^{\times}$to $w^{\times}$.

6. $A=\left(a_{i j}\right)$ is a pairwise additive matrix if $a_{i j}=-a_{j i}$.

"Research supported in part by N̦SERC Canada grant \# A-8802. 
7. $w=\left(w_{k}\right)$ is an additive weight vector if $\sum_{k=1}^{n} w_{k}=0$.

8. $A=\left(a_{i j}\right)$ is an additive consistent matrix if $a_{i j}=w_{i}-w_{j}$ for some additive weight vector $w$.

9. $A^{+}, w^{+}$and $C^{+}$are the sets of all pairwise additive matrices, additive weight vectors and additive consistent matrices, respectively.

10. $f^{+}$is the set of all mappings from $A^{+}$to $w^{+}$.

\subsection{Structure}

1. $A^{\times}, w^{\times}$and $C^{\times}$are all groups under componentwise multiplication; $C^{\times}$is isomorphic to $w^{\times}$, and is a subgroup of $A^{\times}$.

2. $A^{+}, w^{+}$and $C^{+}$are all groups under componentwise addition; $C^{+}$is isomorphic to $w^{+}$, and is a subgroup of $A^{+}$.

3. $A^{\times}, w^{\times}$and $C^{\times}$are isomorphic to $A^{+}, w^{+}$and $C^{+}$, respectively. (The logarithmic function with any fixed basis applied componentwise is an isomorphism and the corresponding exponential function is its inverse.)

\section{The Multiplicative Axioms}

A solution of our problem is a specific mapping $f \in f^{\times}$. The following axioms and theorem provide a characterization of the geometric mean mapping.

Axiom 1 If $A$ is a consistent multiplicative matrix with an underlying multiplicative weight vector $w$ (i.e. $a_{i j}=w_{i} / w_{j}$ and $\prod_{k=1}^{n} w_{k}=$ 1 ), then the solution is the vector $w$, that is, $f(A)=w$.

Axiom 2 The weight $w_{i}$ attributed to alternative $i$ is independent of relative measurements among alternatives other than $i$.

Theorem 1

There is exactly one mapping $w=f(A)$ satisfying Axioms 1-2, namely, the geometric mean, given by

$$
w_{i}=\left(\prod_{j=1}^{n} a_{i j}\right)^{1 / n} .
$$




\section{Properties of the Geometric Mean}

\subsection{Scale Inversion and Independence of Description}

Theorem 2

For any $A \in A^{\times}$, the geometric mean mapping satisfies

$$
f(1 / A)=1 / f(A) .
$$

If the matrix $A$ describes the relative preferences of the decision maker on a ratio scale, then the matrix $1 / A$ describes the same preferences on the inverse ratio scale obtained by replacing $x$ on the original scale with $1 / x$ on the inverse one. It is an elementary principle of the theory of measurement that for $f \in f^{\times}$to be an admissible solution, it must be independent of scale relabelling of this type. Any mapping $f \in f^{\times}$which does not satisfy the independence-of-scale-inversion condition $f(1 / A)=1 / f(A)$ has the unacceptable characteristic that it produces solutions which depend on the description of the problem. Since the eigenvector mapping does not satisfy this condition, it cannot satisfy any set of reasonable axioms for deriving weights from pairwise comparisons.

Relabelling the ratio scale into the inverse ratio scale affects the coding of the input and the output, but it does not affect the preferences stated in either the input or the output of the problem. The process may be described by writing two computer programs which accept the decision maker's description of inconsistent pairwise preferences as input and produce a graph of the weights vector on a one-dimensional scale as output. From the point of view of the decision maker the two programs are identical since he provides a single set of input measurements and receives a single graph as output and is unaware that the two programs code the input and output differently. If these programs use the eigenvector method to compute weights, they will generate inconsistent outputs because the eigenvector method does not satisfy the independence-of-scale-inversion condition (see §6.3).

Note that the decision maker is not required to measure his preferences on the ratio scale and its inverse scale simultaneously but rather to provide a single set of input measurements on a single scale, to be processed by two programs using the same algorithm. Therefore, the philosophical discussion on whether there is or there is not "a natural way for our mind to synthesize its dominance and anti-dominance or recessiveness measures to obtain unified interpretation of reality," and what is "an effective way for dealing with the two sides of human experience" (see [9, p. 192]), is not an acceptable justification for this inconsistency. Similarly, the eigenvector method's scale-inversion dependence cannot be justified as an issue of practice vs. theory (see the first paragraph of $\S 3$ in [10]).

\subsection{Symmetry and Uniqueness}

The transpose of a pairwise multiplicative matrix is its componentwise inverse: $a_{i j}=1 / a_{j i}$ implies $1 / A=A^{T}$. This relationship led Johnson et al. [8] to interpret the violation of the independenceof-scale-inversion condition as the right-left asymmetry of the eigenvector solution. This is also a manifestation of the non-uniqueness of the eigenvector method - if weights can be retrieved through either right or left eigenvectors (Saaty suggests their reciprocals as well in $[10$, p. 158]), which one should be preferred and on what basis? 
In contrast, the geometric mean is symmetric, unique and scale-inversion independent of the description of the problem by Theorem 2 .

\subsection{Independence of Order of Operations - Inter-Level Consistency and Uniqueness}

We demonstrated in $[1,4]$ that the eigenvector method in conjunction with additive normalization of weight vectors and an additive aggregation rule (the weighted arithmetic mean as used in the AHP) lead to different solutions depending on the order of operations. Since there is no intrinsic reason to prefer one order of operations over the other, this inter-level inconsistency results in multiple solutions - another facet of the non-uniqueness of the AHP procedures. In contrast, we proved in [4] that the solution obtained when weights are derived by the geometric mean rule and levels in the hierarchy are combined by the weighted-geometric-mean aggregation rule is unique and independent of order of operations.

\subsection{Algebraic Structure}

As noted in $\$ 2.3$, the underlying mathematical structures are algebraic (rather than analytic). Specifically, we are dealing with groups related by isomorphisms. Not surprisingly in this context, the geometric mean is a homomorphism from $A^{\times}$to $w^{\times}$.

\subsection{Extensibility to the Additive Case, Optimization Models and Error Measurement}

Consider the additive problem of deriving weights from additive pairwise comparison matrices. In the notation of $\$ 2$, we seek a mapping $f \in f^{+}$. All our results for the multiplicative case carry over to the additive case verbatim (see [3,1]). In particular, in complete analogy to the multiplicative case, we can state two additive axioms which are satisfied only by the arithmetic mean $w_{i}=\frac{1}{n} \sum_{j=1}^{n} a_{i j}$ which also satisfies the additive independence-of-scale-inversion condition $f(-A)=-f(A)$. The isomorphisms of $\$ 2.3$ link the arithmetic mean with the geometric mean:

$$
\ln \left\{\left(\prod_{j=1}^{n} e^{a_{i j}}\right)^{1 / n}\right\}=\frac{1}{n} \sum_{j=1}^{n} a_{i j} .
$$

Furthermore, the arithmetic mean is the solution of the optimization problem

$$
\begin{array}{ll}
\min _{w} & \sum_{i=1}^{n} \sum_{j=1}^{n}\left(a_{i j}-\left(w_{i}-w_{j}\right)\right)^{2} \\
\text { s.t. } & \sum_{i=1}^{n} w_{i}=0
\end{array}
$$


naturally associated with the additive problem of specifying $f \in f^{+}$. Similarly, the geometric mean is the solution of the optimization problem

$$
\begin{array}{ll}
\min _{w} & \sum_{i=1}^{n} \sum_{j=1}^{n}\left(\ln a_{i j}-\ln \left(w_{i} / w_{j}\right)\right)^{2} \\
\text { s.t. } & \prod_{i=1}^{n} w_{i}=1, \quad w_{i}>0, \quad i=1, \ldots, n .
\end{array}
$$

The group structures provided within the framework of $\$ 2$ justify the association between the two optimization problems by means of the logarithmic/exponential isomorphisms relating $A^{\times}$and $A^{+}$.

The objective functions of the optimization problems provide measures of consistency in a natural way. In the additive case, this measure is given by $\sum_{i, j=1}^{n} \varepsilon_{i j}^{2}$, where $\varepsilon_{i j}=a_{i j}-\left(w_{i}-w_{j}\right)$ are the error terms. In contrast, the eigenvector solution is not applicable to the additive problem. Elegant as it is, the spectral analysis of positive matrices is not relevant to our decision analysis problem.

\section{Rank Preservation vs. Rank Reversal}

\section{$5.1 m$-step Estimates}

The following theorems explore the notion of comparing alternatives in chains rather than pairwise and demonstrate that an $m$-step estimate of the difference or ratio of the weights of the alternatives can be defined in a natural manner and that this definition is consistent with the arithmetic and geometric means in the additive and multiplicative cases respectively, as well as with the measure of error defined in $\$ 5.4$.

\section{Theorem 3}

Let $A \in A^{+}, m \geq 1$, and $w_{i}=\frac{1}{n} \sum_{k=1}^{n} a_{i k}$. Then

$$
a_{i j}^{(m+1)}=\frac{1}{n^{m}} \sum_{k_{1}, k_{2}, \ldots, k_{m}=1}^{n}\left(a_{i k_{1}}+a_{k_{1} k_{2}}+\cdots+a_{k_{m-1} k_{m}}+a_{k_{m} j}\right)=w_{i}-w_{j} .
$$

If $A \in A^{\times}, m \geq 1$, and $w_{i}=\left(\prod_{k=1}^{n} a_{i k}\right)^{1 / n}$, then

$$
a_{i j}^{(m+1)}=\left(\prod_{k_{1}, k_{2}, \ldots, k_{m}=1}^{n} a_{i k_{1}} \cdot a_{k_{1} k_{2}} \cdot \ldots \cdot a_{k_{m-1} k_{m}} \cdot a_{k_{m} j}\right)^{1 / n^{m}}=\frac{w_{i}}{w_{j}} .
$$

\subsection{Row Dominance Concepts}

We demonstrated in [2] that the claim (see e.g. Saaty and Vargas [11] and Saaty [10]) that the eigenvector solution "preserves rank" is not meaningful. "Strong rank preservation" as defined in [11] is in fact a weak condition. None of the results in [11] is a necessary and sufficient condition for "strong rank preservation." It is an error to attach this property to a certain type of matrices (rather than mappings of matrices) as in the corollary to Theorem 4 in [11] and Saaty and Vargas's 
concept of asymptotically m-dominant matrices has no intrinsic value in the context of our problem. In the paragraph preceding Theorem 5 in [11], Saaty and Vargas state:

"For emphasis, recall from graph theory that an element $a_{i j}^{(m)}$ of $A^{m}$ gives the cumulative dominance of the $i$ th element over the $j$ th element along all chains of length $m$. That is precisely how one measures the consistency relation between that row and each column."

There is no standard notion of "cumulative dominance" in graph theory as implied here (cf. e.g. Busacker and Saaty [7]), and measuring consistency in this particular manner is precisely and circularly equivalent to using the eigenvector method. Finally, the left eigenvector satisfies asymptotic conditions which are completely analogous to the ones atisfied by the right eigenvector.

\subsection{The Eigenvector "Rank Preservation" Examples}

In [11], Saaty and Vargas compare the eigenvector and geometric mean solutions and provide an example for which the geometric mean ranking disagrees with the ranking obtained by the (right) eigenvector which they assume to be correct. We demonstrate in [2] that in fact, Saaty and Vargas's example is a case of the eigenvector solution exhibiting a scale-inversion rank reversal.

\subsection{Belton and Gear's Rank Reversal}

We refer the reader to our detailed study in! [4] of the well-known Belton and Gear's rank reversal phenomenon (see [6]). The main result is that the geometric mean is immune to this type of rank reversal when used in conjunction with the weighted-geometric-mean aggregation rule while the AHP's non-multiplicative procedures - the eigenvector mapping and the weighted-arithmetic-mean aggregation rule - are the source of this controversial feature of the AHP.

\section{Summary and Conclusions}

We established that the geometric mean is the only method for deriving weights from multiplicative pairwise comparisons which satisfies fundamental consistency requirements. It is immune to scaleinversion (or left-right) rank reversal as well as to Belton and Gear's rank reversal. Weights generated by the geometric mean are independent of orders of operations and therefore unique across AHP hierarchies and are independent of the description of the problem. It is also the only solution which preserves the strong algebraic structure of the problem and is naturally (through the logarithmic isomorphism) consistent with the arithmetic mean solution in the additive case.

\section{References}

[1] J. Barzilai, "On the Use of the Eigenvector in the AHP," Proceedings of the Tenth International Conference on Multiple Criteria Decision Making, Taipei, Vol. 1, pp. 291-300, 1992.

[2] J. Barzilai, "Deriving Weights from Pairwise Comparison Matrices," Technical Report, School of Computer Science, Technical University of Nova Scotia, 1996. 
[3] J. Barzilai and B. Golany, "Deriving Weights from Pairwise Comparison Matrices: The Additive Case," Operations Research Letters, 9(6), 407-410, 1990.

[4] J. Barzilai and B. Golany, "AHP Rank Reversal, Normalization and Aggregation Rules," INFOR, 32(2), pp. 57-64, 1994.

[5] J. Barzilai, W.D. Cook and B. Golany, "Consistent Weights for Judgements Matrices of the Relative Importance of Alternatives," Operations Research Letters, 6(3), 131-134, 1987.

[6] V. Belton and T. Gear, "On a Shortcoming of Saaty's Method of Analytic Hierarchies," Omega, 11(3), 228-230, 1983.

[7] R.G. Busacker and T.L. Saaty, Finite Graphs and Networks, McGraw-Hill, 1965.

[8] Johnson, C.R., Beine W.B. and Wang, T!J., A Note on Right-Left Asymmetry in an Eigenvector Ranking Procedure, Journal of Mathematical Psychology, Vol. 19, pp. 61-64, 1979.

[9] T.L. Saaty, The Analytic Hierarchy Process, McGraw-Hill, 1980.

[10] T.L. Saaty, "Eigenvector and Logarithmic Least Squares," European Journal of Operational Research, 48(1), 156-160, 1990.

[11] T.L. Saaty and L.G. Vargas, "Inconsistency and Rank Preservation," Journal of Mathematical Psychology, 28, pp. 205-214, 1984. 